\section{Trombosis venosa profunda y agenesia de vena cava inferior: presentación típica en una anomalía poco frecuente. Caso clínico}

\author{
FERNANDA CASTRO L. ${ }^{1}$, ÁLVARO ZAPICO ${ }^{1}$, RICARDO WILHELM $^{2}$
}

\section{Inferior vena cava agenesis presenting as deep vein thrombosis. Report of one case}

\begin{abstract}
Abnormalities of the inferior vena cava are rare. Its embryological development occurs between the sixth and eighth week of gestation and depends on the persistence or regression of three pairs of veins: the posterior cardinal veins, the subcardinal veins and the supracardinal veins. The type of congenital alteration depends on the moment that embryogenesis is altered. The most frequent clinical presentation is deep vein thrombosis, which occurs mainly in young men. We report a 16-year-old male presenting with edema of the left leg. No risk factor for thrombosis was recorded. A Doppler ultrasound confirmed the presence of a deep femoro-popliteal vein thrombosis. An abdominal CAT scan showed an agenesis of the supra and infra-adrenal inferior vena cava, with multiple collaterals. The study for thrombophilia was negative. The patient was treated with vitamin $K$ antagonists with a good clinical response.
\end{abstract}

(Rev Med Chile 2020; 148: 1833-1837)

Key words: Congenital Abnormalities; Vena Cava, Inferior; Venous Thrombosis.
Servicio de Cirugía Hospital San Martín de Quillota. Quillota,

Chile.

2Servicio de Radiología Hospital San Martín de Quillota. Quillota, Chile.

Los autores declaran no tener conflictos de interés.

Trabajo no recibió financiamiento.

Recibido el 16 de abril de 2020, aceptado el 29 de octubre de 2020.

Correspondencia a: Fernanda Castro Leiva, Servicio de Cirugía General, Hospital San Martín de Quillota. Quillota, Chile. fernanda.cstr@gmail.com

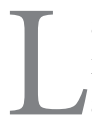

as anomalías congénitas de la vena cava inferior (VCI) son inusuales, siendo la $\checkmark$ agenesia de esta la menos frecuente, con una incidencia aproximada de $0,005 \%$ a $1 \%$ de la población general ${ }^{1,2}$. Las presentaciones más típicas son la insuficiencia venosa crónica, el síndrome de congestión pélvica y la trombosis venosa profunda (TVP) $)^{1,2,6,8,11,12}$. Se describen otros síntomas menos frecuentes, como el dolor lumbar, secundario a la compresión nerviosa causada por el desarrollo de colaterales ${ }^{1,11}$. Sin embargo, generalmente esta anomalía es completamente asintomática.

En esta publicación describiremos el caso clínico de un paciente con TVP asociada a una anomalía poco frecuente de la VCI, y discutiremos la evidencia disponible respecto a la asociación de estas patologías.

\section{Caso clínico}

Hombre de 16 años, sin antecedentes mórbidos, consultó en Unidad de Emergencia por cuadro de 2 semanas de evolución de aumento de volumen y dolor en extremidad inferior derecha, sin otra sintomatología asociada. Sin antecedente de cirugías previas, hábito tabáquico, ni viajes prolongados o trauma en los últimos 30 días.

En el examen físico destacaba aumento de volumen de la extremidad inferior derecha, empastamiento gemelar y signo de Homans positivo. El resto del examen físico no presentaba hallazgos patológicos.

Ante sospecha de trombosis venosa profunda se realizó ecografía Doppler venosa, que confirmó el diagnóstico de TVP fémoro-poplítea derecha.

Como parte del estudio etiopatogénico, se rea- 
lizó una tomografía de abdomen y pelvis contrastada, en la cual se identificó la agenesia de la vena cava inferior supra e infrarrenal, con desarrollo de múltiples colaterales (Figura 1). El estudio de trombofilia fue negativo. Se realizó ecocardiograma para completar estudio de malformaciones asociadas, sin hallazgos patológicos.

Se decidió tratar con medias de compresión graduadas, asociado a heparina de bajo peso molecular, con posterior traslape a antagonistas de vitamina $\mathrm{K}$ y prolongación de tratamiento de manera indefinida. Presentó buena respuesta clínica, con disminución progresiva del edema.

En el seguimiento a 18 meses, el paciente se mantenía asintomático. Sin nuevos episodios de TVP, ni signos de síndrome postrombótico.

El paciente y su representante legal otorgaron su consentimiento para la publicación de datos clínicos.

\section{Discusión}

Las anomalías congénitas de la vena cava inferior son inusuales, siendo la agenesia la menos frecuente, con una incidencia aproximada de $0,005 \%$ a $1 \%$ de la población general ${ }^{1,2}$. Sin embargo, en pacientes jóvenes con diagnóstico de trombosis venosa profunda su incidencia aumenta a $5 \%{ }^{11,12}$.

\section{Anomalías del desarrollo de la vena cava inferior}

El desarrollo embriológico de la vena cava inferior ocurre entre la sexta y octava semana de gestación, y depende de la persistencia o regresión de 3 pares de venas: las venas cardinales posteriores, las venas subcardinales y las venas supracardinales ${ }^{1,3,5,9,10}$ (Figura 2).

El tipo de anomalía varía según el momento del desarrollo embriológico que se ve alterado, de esta manera, la presencia de una VCI izquierda o doble VCI se debe a la persistencia de la vena supracardinal izquierda, acompañado o no de la atrofia de la vena supracardinal derecha ${ }^{3,9}$. Mientras que las anomalías de la vena renal se deben a la persistencia de la vena intersupracardinal, asociada o no a la regresión de la vena intersubcardinal, presentándose en la vida adulta como una vena renal izquierda retroaórtica o una vena renal izquierda circumaórtica ${ }^{3,9}$.

Respecto a la ausencia de VCI, no existe claridad en su etiología, algunos autores han pro-

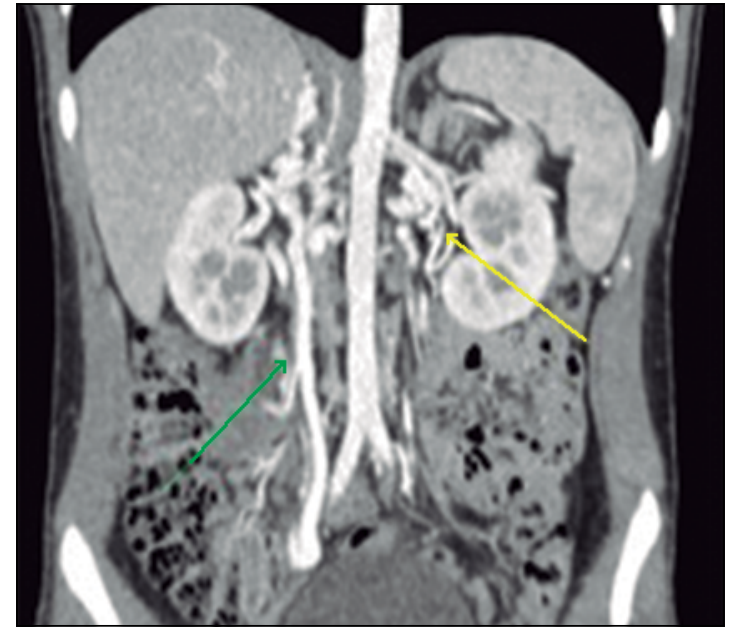

Figura 1. TC de abdomen con contraste, con reconstrucción coronal. Ausencia de $\mathrm{VCl}$ en sus segmentos supra e infrarrenal. Desarrollo de múltiples colaterales paravertebrales (flecha amarilla). Vena testicular derecha dilatada (flecha verde).

puesto un origen secundario a la trombosis de la VCI intrauterina, ocurriendo agenesia de esta y redistribución del retorno venoso en cuatro vías diferentes, las colaterales profundas (paravertebrales y periureterales), colaterales intermedias (gonadales), colaterales superficiales (epigástricas, mamarias internas y subclavias) y vía portal a través de las hemorroidales ${ }^{1}$.

\section{Presentación clínica}

El retorno sanguíneo inadecuado causado por la alteración de la VCI produce hipertensión venosa, a pesar del desarrollo de un suministro colateral considerable, causando estasis venosa que puede conducir a trombosis ${ }^{4,11,12}$. La presencia de embolia pulmonar en pacientes con TVP y atresia de la VCI es rara ${ }^{4,12}$, probablemente porque la migración de trombos es impedida por la extensa red de circulación colateral compensatoria ${ }^{4}$. La ausencia de VCI también puede presentarse como insuficiencia venosa crónica y síndrome de congestión pélvica ${ }^{1,2,4,6,8,11}$. No obstante, la mayoría de los pacientes con agenesia de la IVC son asintomáticos, y el diagnóstico es incidental ${ }^{11}$.

Los pacientes que presentan trombosis venosa profunda en presencia de alteraciones de la VCI suelen presentar dolor lumbar, abdominal o ambos, asociado a edema de extremidades in- 
A
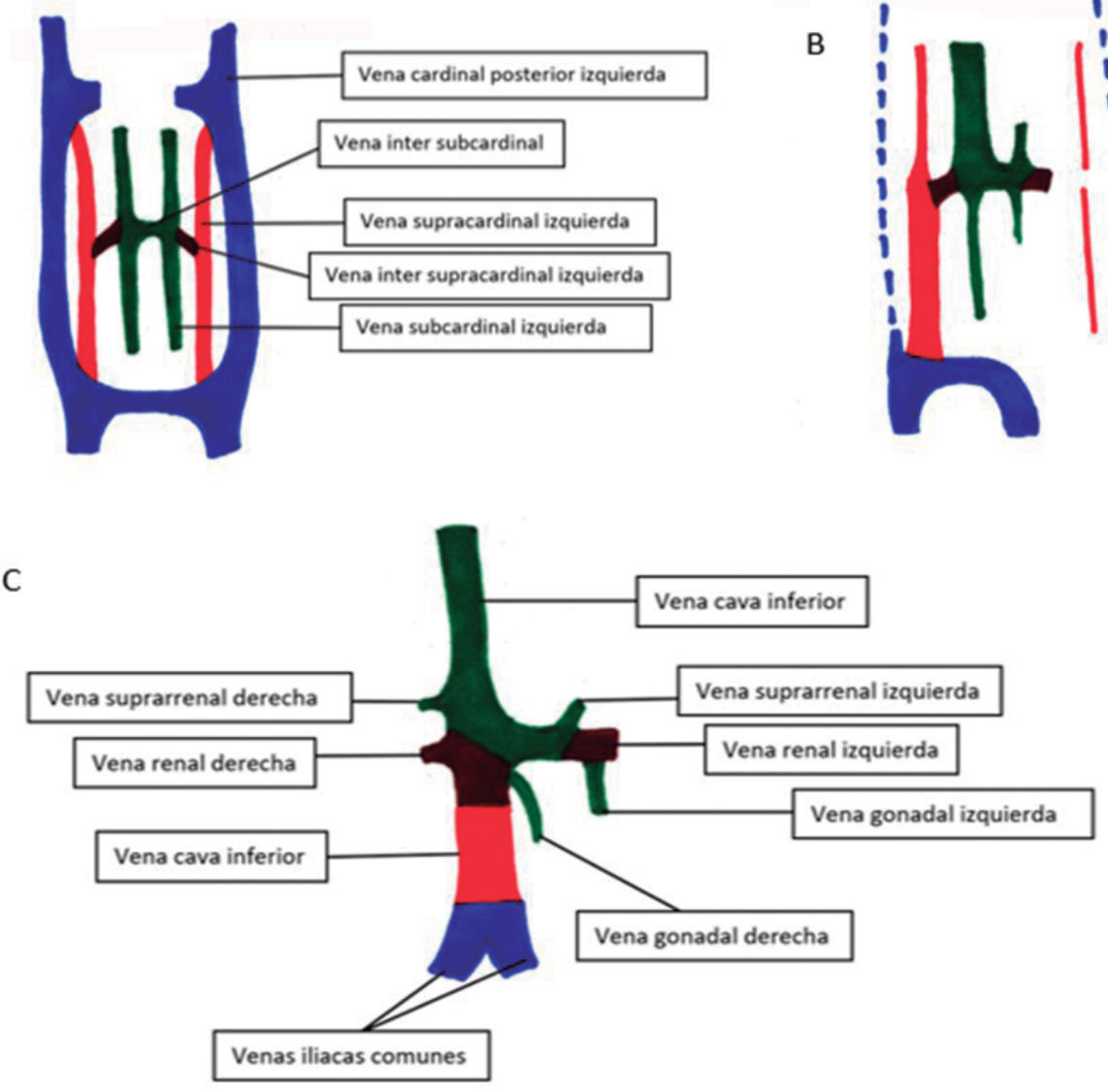

B

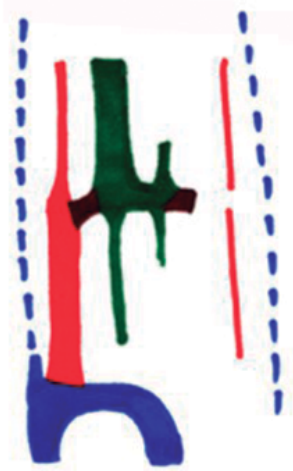

Figura 2. El desarrollo embriológico de la VCl depende de la resistencia o regresión de 3 pares de venas, las venas cardinales posteriores (azul), las venas subcardinales (verde) y las venas supracardinales (rojo). A) Las venas cardinales posteriores son dominantes en la sexta semana de gestación. En la séptima semana comienzan a predominar las venas subcardinales. En la octava semana predominan las venas supracardinales y están formadas las venas intersupracardinales (retroaórtica) e intersubcardinal (preaórtica). B) Las venas izquierdas regresan al igual que la vena cardinal derecha. C) Finalmente, la VCl queda conformada por la vena subcardinal derecha y en su porción infrarrenal por la vena supracardinal derecha. Las venas renales se desarrollan a partir de las venas intersupracardinales.

feriores $^{13}$. La trombosis suele afectar segmentos más distales como la VCI o venas iliacas, y más frecuentemente de manera bilateral ${ }^{8,11,12}$.

Factores de riesgo que pueden desencadenar un estado protrombótico en pacientes con anomalías de la VCI son la inmovilización prolongada, el trauma, las infecciones, las neoplasias, las cirugías, el embarazo y el uso de fármacos protrombóticos, por lo que es importante consignar estos antecedentes en pacientes que cursen con trombosis venosa profunda ${ }^{13}$. Además, se ha descrito en numerosos reportes la asociación de ejercicio intenso y trombosis venosa profunda en pacientes portadores de anomalías congénitas de la $\mathrm{VCI}^{15}$.

\section{Diagnóstico y estudios complementarios}

La ecografía suele ser la primera modalidad de imagen en la evaluación de pacientes con trombosis venosa profunda ${ }^{12}$, sin embargo, las anomalías de la vena cava inferior pueden no ser diagnosticadas en este estudio, por lo que es esencial completar el estudio etiológico en pacientes 
jóvenes, o sin factores de riesgo de trombosis venosa profunda, con una tomografía con contraste de tórax, abdomen y pelvis para la pesquisa tanto de anomalías de VCI como de otros factores de riesgo asociado ${ }^{12,16}$. Otras opciones de estudio diagnóstico de VCI son la resonancia nuclear magnética y la flebografía, aunque estas opciones son menos utilizadas en la actualidad.

Revisiones bibliográficas describen la presencia concomitante de trombofilia en hasta $40 \%$ de los pacientes con alteraciones de la VCI que se presentan con $\mathrm{TVP}^{11,13,14}$, definir la existencia de esta asociación es fundamental para definir el tratamiento a largo plazo de estos pacientes. Por lo tanto, el estudio de estos pacientes siempre debe incluir estudio de trombofilias.

Además, las alteraciones de la VCI pueden asociarse a otras malformaciones como situs inversus, dextrocardia, poliesplenia o asplenia, y en el caso de la agenesia de la VCI, esta puede asociarse a agenesia o hipoplasia renal, configurando el síndrome de $\mathrm{KILT}^{1,6}$, por lo que se recomienda el estudio dirigido a descartar la presencia de estas asociaciones.

\section{Tratamiento}

El tratamiento de las malformaciones de la vena cava inferior dependerá del tipo de alteración y su presentación clínica. En el caso de la trombosis venosa profunda en pacientes con agenesia de VCI, el uso de medias antitrombóticas en conjunto con la anticoagulación oral es considerado actualmente el mejor enfoque ${ }^{1,2,12}$, asociado a minimizar los factores que aumenten el riesgo de $\mathrm{TVP}^{6}$. La duración del tratamiento anticoagulante se debate y no hay evidencia científica clara, algunos autores recomiendan mantenerla 3 meses en casos donde no coexista trombofilia ${ }^{6,12}$. Otro enfoque es su mantención a largo plazo, con el objetivo de disminuir la recurrencia de TVP y la incidencia de síndrome postrombótico ${ }^{11,13}$.

Existen publicaciones que sugieren que la trombólisis dirigida por catéter puede recomendarse como el tratamiento de elección para la TVP iliofemoral aguda en pacientes con atresia de la VCI, ya que este tratamiento puede lograr resultados clínicos satisfactorios, incluida la permeabilidad de las venas profundas ${ }^{11,13}$. Sin embargo, faltan estudios que puedan confirmar el beneficio de esta terapia, versus el tratamiento conservador con anticoagulación, en pacientes con asociación de trombosis venosa profunda y agenesia de VCI.

También se han informado intervenciones quirúrgicas como la trombectomía venosa o incluso reemplazo protésico de la $\mathrm{VCI}$, con resultados exitosos. El enfoque quirúrgico solo se recomienda para aquellos pacientes con deterioro rápido de la insuficiencia venosa crónica o que presentan TVP y colapso concomitante del drenaje venoso colateral $^{1,6,12}$.

\section{Conclusión}

La agenesia de vena cava inferior es una alteración infrecuente, que debe sospecharse en pacientes jóvenes con trombosis de vena cava inferior sin otras causas evidentes, ya que en este grupo es mayor su incidencia. Los métodos de estudios más utilizados son la ecografía Doppler venoso y la tomografía computarizada contrastada. El estudio etiopatogénico siempre debe incluir la pesquisa de malformaciones asociadas y el estudio de trombofilias, debido a su frecuente asociación e importancia en el manejo a largo plazo.

Por lo poco frecuente de esta patología, no existen estudios clínicos con distribución aleatoria de casos que comparen las diversas alternativas de tratamiento, sin embargo, la evidencia existente, aunque limitada por tratarse de reportes de casos retrospectivos, sugiere la eficacia y seguridad del tratamiento con anticoagulación. Aspectos como la duración del tratamiento con anticoagulantes, aún son controversiales en ausencia de trombofilias. La evidencia sugiere una menor incidencia de síndrome postrombótico cuando se realiza trombólisis dirigida por catéter en comparación con anticoagulación de manera exclusiva, sin embargo, hacen falta estudios prospectivos randomizados en el futuro. Métodos terapéuticos más agresivos, como la cirugía, son una alternativa en casos graves o de falta de respuesta a tratamientos más conservadores.

\section{Referencias}

1. Morosetti D, Picchi E, Calcagni A, Lamacchia F, Cavallo A, Bozzi A, et al. Anomalous development of the inferior vena cava: Case reports of agenesis and hypoplasia. Radiol Case Rep 2018; 13 (4): 895-903.

2. Leitão A, Esteves J, Abreu J, Pereira A, Boncoraglio M, 
Certo M, et al. Deep Venous Thrombosis and a Very Rare Finding: Inferior Vena Cava Infra-renal Segment Agenesis. Eur J Case Rep Intern Med 2019; 6(3): 1.

3. González J, Gaynor J, Albéniz L, Ciancio G. Inferior Vena Cava System Anomalies: Surgical Implications. Curr Urol Rep 2017; 18 (2).

4. Khalid M, Nukavarapu M, Shah R, Paul T. Rare case of a kidney and inferior vena cava abnormalities with extensive lower extremity deep vein thrombosis in a young healthy male. Clin Pract 2018; 8 (4): 1079.

5. Shin D, Sandstrom C, Ingraham C, Monroe E, Johnson $\mathrm{G}$. The inferior vena cava: a pictorial review of embryology, anatomy, pathology, and interventions. Abdom Radiol (NY) 2019; 44 (7): 2511-27.

6. Ramos Aranda J, Ramírez Cerda C, Cohen Mussali S, Valdés Flores J. Inferior Vena Cava Agenesis: An Unusual Cause of Deep Vein Thrombosis and Pulmonary Embolism in Young Adult Patients. EJVES Short Rep 2018; 39: 12-5.

7. Menezes T, Haider E, Al-Douri F, El-Khodary M, Al-Salmi I. Pelvic congestion syndrome due to agenesis of the infrarenal inferior vena cava. Radiol Case Rep 2019; 14 (1): 36-40.

8. Langer F, Dos Santos D, Suertegaray G, Pereira Haygert C. Bilateral Deep Vein Thrombosis Associated with Inferior Vena Cava Agenesis in a Young Patient Manifesting as Low Back Pain. Acta Med Port 2017; 30 (4): 333.

9. Morato A, Quiroz O, Ramírez J. Anomalías congénitas de la vena cava inferior. El rol de la tomografía computada multidetector. An Radiol Mex 2012; 2: 104-13.

10. Motta-Ramírez G, Mundo-Gómez C, Ramírez-Arias J.
La vena cava y sus variantes anatómicas. Rev Mex Angiol 2010; 38: 19-29.

11. Broholm R, Jørgensen M, Just S, Jensen L, Bækgaard N. Acute Iliofemoral Venous Thrombosis in Patients with Atresia of the Inferior Vena Cava Can Be Treated Successfully with Catheter-directed Thrombolysis. J Vasc Interv Radiol 2011; 22 (6): 801-5. doi: 10.1016/j. jvir.2011.01.449.

12. Gayer G, Luboshitz J, Hertz M, Zissin R, Thaler M, Lubetsky A, et al. Congenital anomalies of the inferior vena cava revealed on CT in patients with deep vein thrombosis. AJR Am J Roentgenol 2003; 180 (3): 72932.

13. Pozzi A, El Lakis MA, Chamieh J, Carbonell BB, Villa F. The Typical Presentation Spectrum of Deep Vein Thrombosis Associated with Inferior Vena Cava Malformations. Thrombosis 2016; 2016: 4965458. doi: 10.1155/2016/4965458.

14. Sagban T, Scharf R, Wagenhäuser M, Oberhuber A, Schelzig H, Grabitz K, et al. Elevated risk of thrombophilia in agenesis of the vena cava as a factor for deep vein thrombosis. Orphanet J Rare Dis 2015; 10: 3 . Retrieved 20 September 2020.

15. García-Fuster M, Forner M, Flor-Lorente B, Soler J, Campos S. Anomalías de la vena cava y trombosis venosa profunda. Rev Esp Cardiol 2006; 59 (2): 171-5 Retrieved 20 September 2020.

16. Lambert M, Marboeuf P, Midulla M, Trillot N, Beregi JP, Mounier-Vehier $C$, et al. Inferior vena cava agenesis and deep vein thrombosis: 10 patients and review of the literature. Vasc Med 2010; 15 (6): 451-9. doi: $10.1177 / 1358863 \times 10391355$. 